\title{
Selective Electrical Stimulation of the Auditory Nerve Activates a Pathway Specialized for High Temporal Acuity
}

\author{
John C. Middlebrooks ${ }^{1,2}$ and Russell L. Snyder ${ }^{3,4}$ \\ ${ }^{1}$ Kresge Hearing Research Institute, University of Michigan, Ann Arbor, Michigan 48109, ${ }^{2}$ Departments of Otolaryngology, Neurobiology and Behavior, \\ and Cognitive Science, University of California at Irvine, Irvine, California 92697-5310, ${ }^{3}$ Epstein Laboratory, Department of Otolaryngology, University of \\ California at San Francisco San Francisco, California 94143-0526, and ${ }^{4}$ Department of Psychology, Utah State University, Logan, Utah 84322
}

Deaf people who use cochlear implants show surprisingly poor sensitivity to the temporal fine structure of sounds. One possible reason is that conventional cochlear implants cannot activate selectively the auditory-nerve fibers having low characteristic frequencies (CFs), which, in normal hearing, phase lock to stimulus fine structure. Recently, we tested in animals an alternative mode of auditory prosthesis using penetrating auditory-nerve electrodes that permit frequency-specific excitation in all frequency regions. We present here measures of temporal transmission through the auditory brainstem, from pulse trains presented with various auditory-nerve electrodes to phaselocked activity of neurons in the central nucleus of the inferior colliculus (ICC). On average, intraneural stimulation resulted in significant ICC phase locking at higher pulse rates (i.e., higher "limiting rates") than did cochlear-implant stimulation. That could be attributed, however, to the larger percentage of low-CF neurons activated selectively by intraneural stimulation. Most ICC neurons with limiting rates $>500$ pulses per second had CFs $<1.5 \mathrm{kHz}$, whereas neurons with lower limiting rates tended to have higher CFs. High limiting rates also correlated strongly with short first-spike latencies. It follows that short latencies correlated significantly with low CFs, opposite to the correlation observed with acoustical stimulation. These electrical-stimulation results reveal a high-temporal-acuity brainstem pathway characterized by low CFs, short latencies, and high-fidelity transmission of periodic stimulation. Frequency-specific stimulation of that pathway by intraneural stimulation might improve temporal acuity in human users of a future auditory prosthesis, which in turn might improve musical pitch perception and speech reception in noise.

\section{Introduction}

The temporal fine structure of a sound is the cycle-by-cycle timing of the waveform. In normal hearing, transmission of fine structure to the brain is subject to temporal imprecision introduced by cochlear mechanics, transduction, and transmission at the hair-cell synapse. Electrical stimulation of the auditory nerve through cochlear implants bypasses that imprecision. Indeed, auditory nerve fibers phase lock to higher frequencies when stimulated electrically than when stimulated with sound (van den Honert and Stypulkowski, 1987; Hartmann and Klinke, 1990; Javel, 1990). For those reasons, one might hypothesize that temporal perception should be more acute in electrical hearing than in normal hearing. Contrary to that hypothesis, cochlear-implant users show poorer sensitivity to temporal fine structure than do normal-hearing listeners. For example, implant users generally report sensations of increasing pitch corresponding to increasing electrical pulse rates only up to $\sim 300$ pulses per second (pps) (Zeng, 2002), whereas normal-hearing listeners perceive increasing temporal pitch up to $\sim 2000 \mathrm{~Hz}$ (Moore, 1973). Moreover,

Received 0ct. 5, 2009; revised Dec. 8, 2009; accepted Dec. 13, 2009.

This work was supported by National Institutes of Health Grants N01-DC50005 and P30 DC05188. We thank Jim Wiler for expert technical support, Zekiye Onsan for help with the illustrations, and Chen-Chung Lee and Alana Kirby for assistance in the experiments.

Correspondence should be addressed to John C. Middlebrooks, Department of 0tolaryngology, 404D Med Sci D., University of California, Irvine, Irvine, CA 92697-5310. E-mail: j.midd@uci.edu.

DOI:10.1523/JNEUROSCI.4949-09.2010

Copyright $\odot 2010$ the authors $\quad 0270-6474 / 10 / 301937-10 \$ 15.00 / 0$ bilateral implant users lose sensitivity to interaural time differences at pulse trains above $100 \mathrm{~Hz}$, whereas normal-hearing listeners show no such impairment at high stimulus rates.

Recently, we tested in animals the feasibility of direct auditory nerve stimulation with a penetrating electrode array as an alternative to a conventional intrascalar cochlear implant (Middlebrooks and Snyder, 2007, 2008). Compared with cochlear-implant stimulation, intraneural stimulation produced lower thresholds, more restricted spread of excitation along the frequency axis, and more selective excitation of low-frequency fibers from the cochlear apex. Intraneural stimulation also showed shorter temporal integration time constants, consistent with spike initiation in auditory nerve axons, whereas stimulation with cochlear implants yielded time constants consistent with initiation in cell bodies or dendrites (Middlebrooks and Snyder, 2007). Based on these results, we hypothesized that the relatively intimate contact between intraneural electrodes and nerve fibers might enhance transmission of temporal fine structure.

We tested this hypothesis in anesthetized cats by stimulating the auditory nerve with intraneural and intrascalar electrodes and recording from neurons in the central nucleus of the inferior colliculus (ICC). We quantified transmission of temporal fine structure by measuring the maximum stimulus pulse rates to which ICC neurons showed significant phase locking: their limiting rates. Consistent with our hypothesis, limiting rates averaged higher in the intraneural-stimulation condition. Additional analysis, however, demonstrated a robust correlation of high lim- 
iting rates with low characteristic frequencies (CFs) of ICC neurons. Within any particular range of CF, ICC neurons showed similar limiting rates, regardless of mode of nerve stimulation. The higher limiting rate distribution observed with intraneural stimulation could be accounted for by the larger percentage of low-CF neurons that were sampled. Neurons with high limiting rates also tended to have shorter latencies to electrical stimulation. The results demonstrate the presence of a high-temporalacuity pathway characterized by short latencies, low CFs, and high limiting rates. Selective stimulation of that pathway could enhance temporal acuity in users of a future auditory prosthesis using intraneural stimulation, resulting in improvements in $\mathrm{mu}-$ sical pitch perception and improved speech perception in noise.

\section{Materials and Methods}

Experiments were conducted at the University of Michigan with approval of the University of Michigan Committee on Use and Care of Animals. Procedures for acoustical and electrical stimulation and for multisite recording from the inferior colliculus were similar to those described in detail previously (Middlebrooks and Snyder, 2007) and will be summarized here.

Data presented were from experiments in five barbiturate-anesthetized cats. Each experiment involved the following steps. (1) A 32-site silicon-substrate recording probe was implanted in the right ICC, oriented in the coronal plane at $35^{\circ}$ from the sagittal plane, approximately parallel to the tonotopic axis of the ICC of increasing CF. (2) Based on the responses to tones presented to the left (contralateral) ear through a calibrated ear bar, the recording probe was adjusted in depth to sample CFs from $<1$ to $>32 \mathrm{kHz}$, and then the probe was fixed in place. (3) Frequency response areas measured with pure tones were obtained with $1 / 6$ octave resolution in frequency and $5 \mathrm{~dB}$ resolution in sound level. The frequency response areas yielded a CF for each recording site, which was used in later interpretation of responses to electrical stimulation. (4) The right ear was deafened by removal of the tympanic membrane and ossicles, resulting in a severe conductive hearing loss. The left ear was deafened by injection of neomycin sulfate ( $10 \%$ in water) into the scala tympani. In pilot experiments in other animals, that procedure consistently led to $>80 \mathrm{~dB}$ elevations of auditory thresholds (i.e., loss of all scalp-recorded auditory brainstem response at levels within the dynamic range of our earphones) within $\sim 10 \mathrm{~min}$. (5) ICC spike activity was recorded in response to single electric pulses and to pulse trains presented through each of three types of stimulating electrode. First, a siliconsubstrate intraneural stimulating array was inserted into the auditory nerve through a small hole in the osseous spiral lamina for intraneural stimulation. Second, an eight-channel banded electrode array (Cochlear Ltd.) was inserted in the scala tympani through a small cochleostomy for intrascalar stimulation. Third, the apex of the cochlea was exposed, and a silver apical ball electrode was placed on the osseous spiral lamina of the apical turn. The apical ball electrode was intended to simulate an intrascalar electrode advanced all the way to the extreme apex of the cochlea, which is impossible with present-day cochlear implants. The duration of experiments, from induction of anesthesia to termination, ranged from 18 to $25 \mathrm{~h}$, typically with only minor reductions in responsiveness observed.

Stimulus presentation and data acquisition used System 3 equipment from Tucker Davis Technologies (TDT) and custom software running in MATLAB (MathWorks). Each recording probe (NeuroNexus Technologies) had 32 iridium-plated recording sites spaced in $100 \mu \mathrm{m}$ intervals along a single silicon-substrate shank. The 32 recorded neuronal waveforms were digitized simultaneously, displayed online, and stored on computer disk for offline spike sorting. Electrical artifact from the cochlear stimulus could be detected at the ICC recording sites. Artifact was eliminated by a sample-and-hold function that was programmed into the digital signal processor in the TDT recording system, effectively gating off the amplifier during the brief times of the stimulus pulses (Middlebrooks, 2008). In a few instances in which adequate artifact rejection could not be attained and in test cases in which the artifact rejection was disabled, artifact propagated to the ICC with group delay of $<2 \mathrm{~ms}$. Such short-delay activity was distinct from spike activity (with group delay $>4 \mathrm{~ms}$ ); recordings were screened to eliminate units in which recordings were contaminated by electrical artifact.

Neural spikes were detected offline using a spike-sorting procedure described previously (Middlebrooks, 2008). Among the five animals, 2-4 of the 32 sites on each probe yielded well isolated single units, and the remaining 28-30 sites each yielded unresolved activity from two or more neurons. We refer to the spike activity at all recording sites as "unit" activity except in cases in which we specifically refer to "well isolated single units." Activity was recorded from a total of 160 sites (i.e., 32 in each of five cats). The number of units included in specific analyses varied according to the numbers of ICC units that were activated by the various stimuli.

Three types of stimulating electrodes or electrode arrays were used. Intraneural stimulating arrays were NeuroNexus probes similar to the recording probes. Each stimulating array had 16 iridium-plated electrodes centered at $100 \mu \mathrm{m}$ intervals along a single silicon shank. The shank was inserted into the auditory nerve through a hole in the osseous spiral lamina, approximately perpendicular to the trunk of the auditory nerve as it exited the base of the cochlea. Based on the tonotopic distribution of ICC activation, we selected two (in two animals) or three (in three animals) intraneural stimulating electrodes to represent activation of auditory-nerve fibers from basal, middle, and/or apical turns. The intrascalar electrode arrays were similar to the clinical Nucleus 22 cochlear implant, differing from the human version only in that the array was truncated to eight electrodes. In each animal, the intrascalar array was advanced as far apically as possible, typically lying with the most apical electrode in the basal half of the middle turn. The most apical stimulating electrodes (designated electrodes 7 and 8) exhibited the lowest thresholds, presumably because of their snug fit in the scala tympani. For monopolar stimulation, we used electrode 7 or 8 as the active electrode and a wire in a neck muscle as the return. For bipolar stimulation, electrode 7 was the active electrode and electrode 8 was the return. The apical ball electrode was a $\sim 0.25 \mathrm{~mm}$ ball flamed on the end of a silver wire. The ball was positioned on the exposed osseous spiral lamina of the apical turn of the cochlea.

Custom optically isolated 8- or 16-channel current sources were used for electrical stimulation. Output impedances were $\sim 2 \mathrm{M} \Omega$ for intraneural electrodes and $\sim 20 \mathrm{k} \Omega$ for the intrascalar and apical ball electrodes. Current pulses were biphasic, initially cathodal, $40 \mu$ s per phase, with no interphase gap. Single pulses were used to derive plots of the tonotopic distribution of ICC activation as a function of current level, known as spatial tuning curves. Measures of transmission of temporal fine structure used pulse trains, $300 \mathrm{~ms}$ in duration, repeated every $600 \mathrm{~ms}$. Pulse rates ranged from 39.99 to $602.82 \mathrm{pps}$ in steps of $38.52-41.79 \mathrm{pps}$; hereafter, the rates will be written as $40-600 \mathrm{pps}$ in 40 pps steps for convenience of presentation. Current levels of the pulse trains were 4 and $8 \mathrm{~dB}$ above the estimated single-pulse detection threshold of units at the most sensitive ICC recording site; in some cases, additional levels at $2 \mathrm{~dB}$ increments were tested. Each combination of pulse rate and level was tested 20 times in interleaved order.

Thresholds for detection of single pulses were computed based on trial-by-trial spike counts using a procedure derived from signal detection theory (Green and Swets, 1966; Macmillan and Creelman, 2005; Middlebrooks and Snyder, 2007). Background (i.e., no stimulus) spike counts were measured in the intervals 18 to $3 \mathrm{~ms}$ before the pulse, and stimulus-driven spike counts were measured in the intervals 3 to $18 \mathrm{~ms}$ after the pulse. For each stimulus level, we formed an empirical receiver operating characteristic (ROC) curve based on the trial-by-trial distributions of background and driven spike counts. The area under the ROC curve gave the probability of correct detection, which was expressed as a $z$-score and was multiplied by $\sqrt{ } 2$ to obtain the detection index, $d^{\prime}$.

Analysis of the responses of each unit to pulse trains was conducted at the lowest tested level at which $d^{\prime}$ for that unit was $\geq 1$. That relatively low level was favored because many units could not be tested at higher levels, either because of the greater incidence of electrical artifact at higher levels or because the stimulator could not generate sufficiently high currents to stimulate some high-threshold units at high suprathreshold levels. 


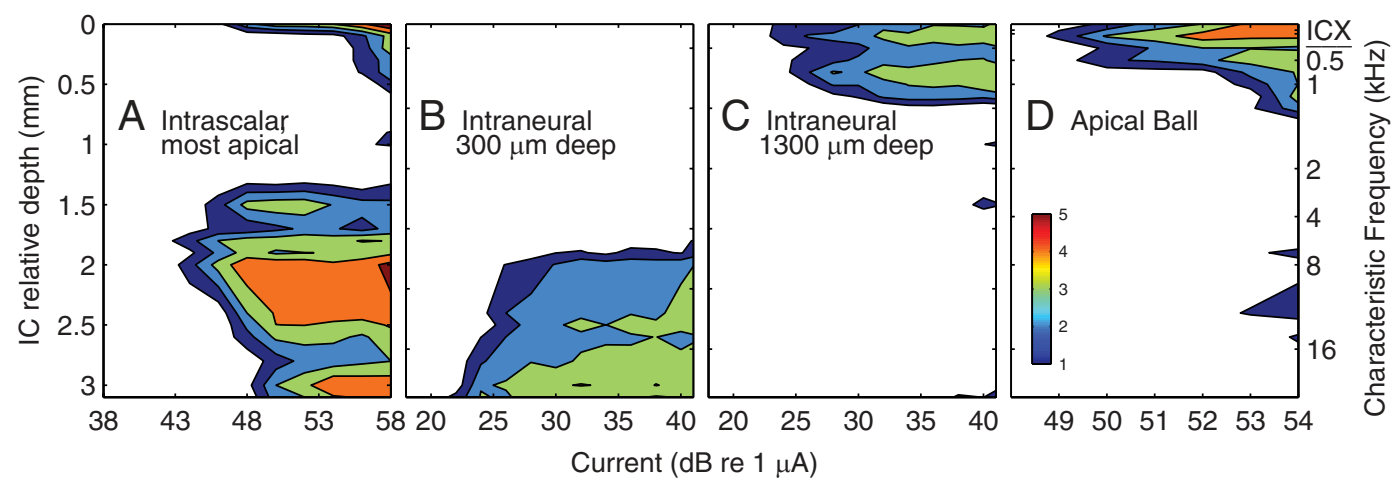

Figure 1. Distributions of inferior colliculus (IC) activity elicited by single electrical pulses from various electrodes in a single animal. Each panel shows IC activity as a function of depth along the recording probe (ordinate) and current level (abscissa). The right axis indicates characteristic frequencies at various IC depths, determined by tests with tonal stimuli before deafening the animal. In this animal, the three most superficial recording sites were in the ICX. All others were in the ICC. The filled contours represent discrimination index ( $\left.d^{\prime}\right)$ for discrimination of increasing current level based on neural spike rates (as described in Materials and Methods); contours are indicated in steps of $1 d^{\prime}$ unit. Panels differ by cochlear stimulating electrode. $A$, The most apical of the electrodes on an intrascalar cochlear implant. $\boldsymbol{B}, \boldsymbol{C}$, Two intraneural electrodes, with depths in the nerve indicated relative to the most superficial electrode. $\boldsymbol{D}, \mathrm{A}$ ball electrode placed on the osseous spiral lamina of the apical cochlear turn.

Eleven of the 160 recording sites were judged in offline analysis to be in the external nucleus of the inferior colliculus (ICX) on the basis of broad frequency tuning and electrically evoked first-spike latencies longer than $10 \mathrm{~ms}$. In the intraneural condition, units at some of the 149 ICC recording sites were counted two or three times because they were tested with intraneural electrodes at two or three depths in the nerve; for that reason, the number of units sampled could exceed the number of physical recording sites. Sample sizes at the minimum suprathreshold level were 170, 100, 90, and 83 ICC units tested with intraneural, monopolar, bipolar, and apical ball configurations, respectively. Whenever possible, the analysis was repeated at an additional level 2 or $4 \mathrm{~dB}$ higher than the minimum. Sample sizes at the higher level were 121, 47, 44, and 18 sites, respectively. In most cases, results obtained at the low current level were confirmed at the higher levels, as indicated in text, although the statistical power often was lower at the higher levels because of the lower sample size or the reduced range of CFs that was sampled.

The strength of phase locking of ICC neurons to electrical pulse trains was represented by the vector strength (Goldberg and Brown, 1969), which was computed as follows. Each spike was treated as a vector of unit length and of orientation given by its phase relative to the stimulus period. Spikes were summed across 20 trials. The length of the resultant vector divided by the spike count gave the vector strength (possibly ranging from 0 to 1 ), and the orientation of the resultant gave the mean phase. The statistical significance of the vector strengths were evaluated by the Rayleigh test of circular uniformity (Mardia, 1972) at the level of $p<$ 0.001 . The limiting rate for each unit was the highest pulse rate at which the vector strength was statistically significant (i.e., at which the vector strength was greater than the Rayleigh criterion). When units phase locked significantly at the highest tested rate, the limiting rate was scored as $600 \mathrm{pps}$, but we assume that the actual limiting rates of most or all of those units were higher. Mean phase lag tended to increase linearly with increasing stimulus pulse rate across the range of rates that displayed significant phase locking. The slope of the best-fitting phase-versusfrequency line gave the group delay, $D=\Delta \varphi / 2 \pi \Delta r$, for mean phase lag $(\varphi)$ in radians and pulse rate $(r)$ in s $^{-1}$. Units in the ICC responded to the onset of a pulse train with a temporally compact burst of spikes, regardless of the pulse rate, which could have given an erroneous impression of precise phase locking. For that reason, vector strength and mean phase were computed based on spikes falling 50-300 ms after the onset of the pulse train. The same poststimulus time interval was used for measurement of tonic spike rate.

\section{Results}

\section{Spread of ICC activation}

We stimulated the auditory nerve using a variety of electrode configurations, including intrascalar electrodes in monopolar and bipolar configurations, intraneural electrodes, and an apical ball electrode. These configurations varied considerably in the locations and breadths of the activity patterns that they elicited along the ICC tonotopic axis. Examples from one animal are shown in Figure 1. Spatial tuning curves show the spread of ICC activation along the tonotopic axis (ordinate) as a function of current level (abscissa); note that the current scales vary among panels. The three most apical recording sites in this animal were judged to be in the ICX on the basis of broad frequency tuning that was encountered with tonal stimulation. The most apical intrascalar stimulating electrode (Fig. $1 A$ ) in this animal, stimulated in monopolar configuration, activated the ventral half the ICC that was sampled, corresponding to CFs from $\sim 3$ to $32 \mathrm{kHz}$, as well as dorsally located units that were judged to be in the ICX. This was one of three animals in which the most apical intrascalar electrode activated units with CFs ranging from $\sim 2-4 \mathrm{kHz}$ up to $32 \mathrm{kHz}$ or higher. In the two other animals, activity spread more broadly such that units at all ICC recording sites were activated with thresholds within a $5 \mathrm{~dB}$ range. Bipolar intrascalar stimulation (data not shown) in all the tested animals showed somewhat more restricted spread of activation, with little or no spread to dorsal low-CF ICC regions and less spread to the highest-CF regions. Intraneural stimulation produced patterns of activation that varied according to the location of the electrode in the auditory nerve, as described previously (Middlebrooks and Snyder, 2007, 2008). The illustration shows examples of high-CF (Fig. $1 B$ ) and low-CF (Fig. 1C) activation. Note the lower thresholds for intraneural stimulation compared with the monopolar intrascalar and apical ball cases.

Intraneural electrodes activated low-CF regions of the ICC that could not be activated selectively by intrascalar electrodes in the cat. We wanted to compare a form of intrascalar stimulation of low-CF pathways with low-frequency intraneural stimulation. For that reason, we exposed the cochlear apex in each animal and stimulated with a monopolar ball electrode placed on the osseous spiral lamina. In each animal, the apical ball electrode produced restricted low-CF stimulation, as in Figure $1 D$. This is one of three animals in which activation remained restricted to the low-CF region of the ICC across the 5-7 dB range above minimum threshold that was tested. In the two other animals, activation was restricted to low-CF ICC regions only at low levels and spread broadly along the ICC tonotopic axis when stimulus levels were 4 or $6 \mathrm{~dB}$ above the minimum threshold. Although the limited spread of activation produced by stimulation with the 
apical ball electrode resembled that obtained with intraneural stimulation, the high threshold and narrow dynamic range were more like that obtained with conventional intrascalar electrodes.

Phase locking to stimulus fine structure Neurons in the ICC showed robust phase locking to stimulation of the auditory nerve with electrical pulse trains. Examples of phase locking in response to intraneural stimulation are shown in Figure 2 for one well isolated single unit. Left and right columns show poststimulus time histograms (Fig. 2A) and period histograms (Fig. $2 B$ ); stimulus pulse rates are indicated along the left axis. This unit was typical in that it responded reliably to the onset of the pulse train at all pulse rates. At the lower pulse rates, tonic responses phase locked to the stimulus pulses are evident throughout the $300 \mathrm{~ms}$ duration of the pulse train, whereas the tonic response was attenuated at the higher rates. The period histograms show that, at each pulse rate, spikes tended to fall within a restricted portion of the stimulus period. This is most evident at pulse rates up to 360 pps in this example, but a significant number of spikes fell around a particular phase even at 600 pps. The arrowheads in Figure $2 B$ indicate the mean phase computed by vector analysis of spike patterns.

Figure 3 summarizes the pulse-rate dependence of the unit shown in Figure 2. In this example, vector strength, a measure of the strength of phase locking, declined gradually up to $400 \mathrm{pps}$ and varied around low levels at higher pulse rates (Fig. $3 A$ ). Nevertheless, vector strength remained above the Rayleigh criterion for statistically significant phase locking (indicated by the dashed line) across the entire range of tested pulse rates. For this unit, therefore, the "limiting rate" was 600 pps or higher. This unit was typical of most units in that its phase locking exhibited a low-pass dependence on pulse rate, i.e., the vector strength primarily decreased as a function of increasing pulse rate. Bandpass pulse-rate dependence was seen in only $8.6,1.0,4.4$, and $2.3 \%$ of units when stimulated with intraneural, monopolar intrascalar, bipolar intrascalar, or apical ball electrodes, respectively.

The mean phases of responses (Fig. $3 B$ ) increased linearly across the range of tested pulse rates, further supporting the conclusion that phase locking of this unit was significant up to at least 600 pps. In the cases of other units with lower limiting rates, the mean-phase-versus-pulse-rate plot tended to deviate markedly from the linear slope at pulse rates above the limiting rate. For the illustrated unit, the group delay from electrical stimulus to ICC response, calculated from the slope of the phase-versus-pulserate line, was $4.7 \mathrm{~ms}$.

The tonic spike rate (Fig. 3C), counted in the interval from 50 to $300 \mathrm{~ms}$ after pulse-train onset, showed a strong tonic discharge at low pulse rates, beginning to decline at pulse rates above 320 pps. The tonic spike rates of all units showed a low-pass or, as in this example, a weakly bandpass dependence on stimulus pulse rate. We presume that we would have observed a higher percent- age of bandpass rate responses had we consistently tested stimulus pulse rates below 40 pps.

The distribution of limiting rates across the sampled ICC population varied substantially among stimulation configurations. Cumulative distributions are shown in Figure 4. The distributions of limiting rates for monopolar and bipolar intrascalar stimulation were very similar, with median values of 120 pps. Intraneural and apical ball stimulation produced phase locking extending to higher rates, with median values of 200 and 241 pps, respectively. The median limiting rate for intraneural stimulation was even higher when the computation was limited to the stimulating electrode tested in each animal that produced the strongest low-CF activation; that median limiting rate was 281 pps (data not shown). Phase locking to monopolar and bipolar intrascalar stimulation was nearly absent at 600 pps, whereas 13$14 \%$ of ICC units phase locked significantly to 600 pps pulse trains through the intraneural or apical ball electrodes.

The distribution of limiting rates in Figure 4 was based on response of each unit at the lowest suprathreshold current level that was tested. The same analysis based on current levels 2 or 4 $\mathrm{dB}$ higher yielded median limiting rates that were higher for each stimulus configuration: 400, 220, 200, and 561 pps for intraneural, monopolar, bipolar, and apical ball stimulation, respectively.

Tonic firing rates tended to decrease monotonically with increasing stimulus pulse rate, as shown in the example in Figure $3 C$. Figure $5 A$ shows the medians and interquartile ranges of tonic firing rates across our sample of ICC responses to intra- 

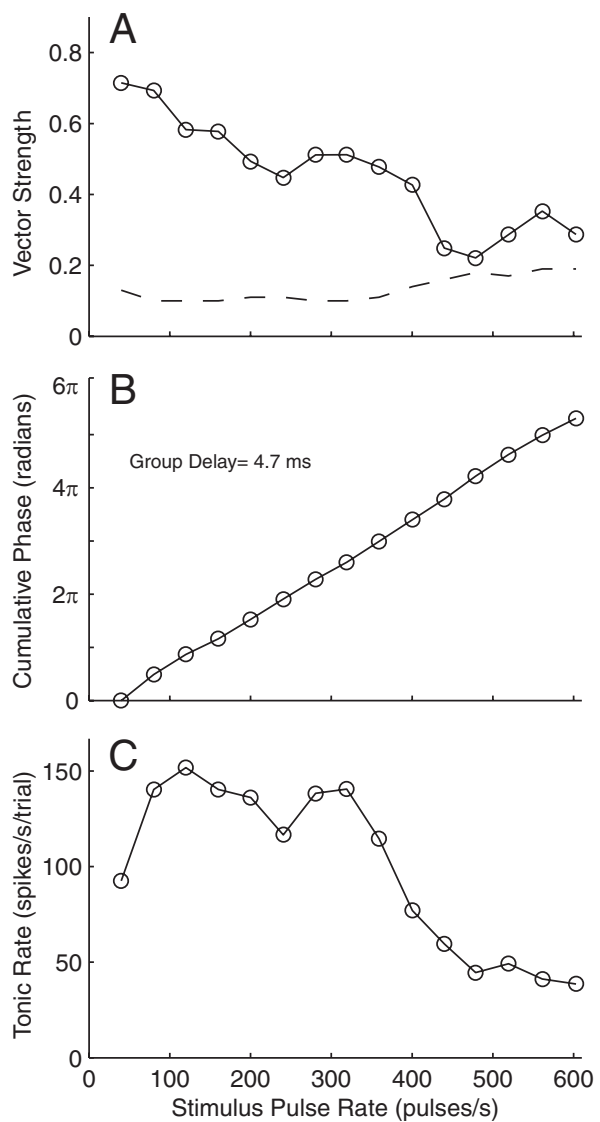

Figure 3. Pulse-rate dependence of responses shown in Figure 2. A, Vector strength (solid line and symbols) as a function of pulse rate. The dashed line represents the Rayleigh criterion for statistically significant phase locking at a level of $p<0.001$. B, Cumulative mean phase lag. The group delay was computed from the slope of this phase-versus-pulse rate line as described in Materials and Methods. C, Tonic rate. Spike rate was computed from responses in the interval $50-300$ ms after stimulus onset.

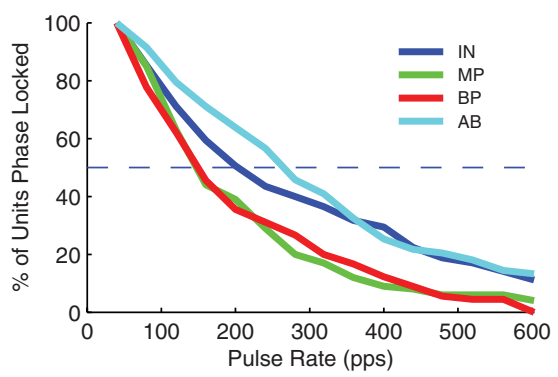

Figure 4. Cumulative distribution of limiting rates of ICC units. Lines represent the percentage of ICC units showing limiting rates at or above each pulse rate. Colors represent various electrode types: IN, intraneural; MP, monopolar intrascalar; BP, bipolar intrascalar; $A B$, apical ball.

neural stimulation; the firing rate for each unit is normalized according to its response to the $40 \mathrm{pps}$ pulse rate. Tonic rates declined to $\sim 10 \%$ at $300 \mathrm{pps}$ and to $\sim 5 \%$ at $600 \mathrm{pps}$. The median firing rates for the various stimulus configurations are compared in Figure $5 B$. The effect of configuration on tonic firing paralleled the effect on limiting rates for phase locking. Intraneural and apical ball stimulation consistently produced tonic firing to higher pulse rates than did monopolar and bipolar intrascalar stimulation. For instance, median tonic rates in response to intraneural stimulation in the range of $80-600$ pps were $12-48 \%$

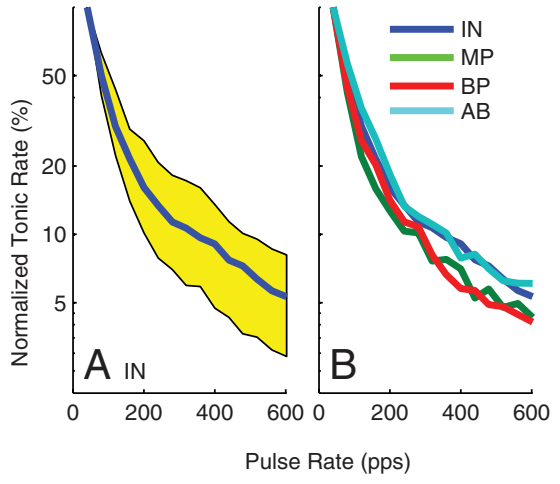

Figure 5. Normalized tonic firing rates as a function of stimulus pulse rate. Tonic firing rates were computed for the interval $50-300 \mathrm{~ms}$ after stimulus onset and normalized by the rate at $40 \mathrm{pps}$. $A$, Median (line) and interquartile range (shading) of normalized tonic rates elicited by intraneural stimulation. $\boldsymbol{B}$, Median normalized tonic rates elicited by the various electrode types indicated by colors. IN, Intraneural; MP, monopolar intrascalar; BP, bipolar intrascalar; $\mathrm{AB}$, apical ball.

higher (mean of $26.5 \%$ ) than in response to monopolar stimulation. Stimulus levels 2 or $4 \mathrm{~dB}$ higher than the minimum suprathreshold level accentuated the effect of configuration on tonic firing: the tonic firing rate for intraneural stimulation averaged $45.9 \%$ higher than that for monopolar stimulation. Note that the low-pass pulse rate sensitivity of tonic firing rates obtained in electrical-stimulation conditions contrasts with the bandpass sensitivity that often is encountered using amplitude-modulated acoustical stimuli (Langner and Schreiner, 1988).

The various stimulation configurations differed in the overall distribution of ICC tonotopic regions that were stimulated. In particular, the intraneural and apical ball electrodes engaged apical-turn (low-frequency) fibers that could not be stimulated selectively by the basally located (high-frequency) intrascalar electrode arrays. We tested the hypothesis that the differences in the ranges of limiting rates observed among stimulation configurations might reflect a relationship between CF and limiting rate. That hypothesis is supported by plots of limiting rates as a function of the CFs of ICC units (Fig. 6). As expected, relatively few units with CFs $<1.5 \mathrm{kHz}$ could be stimulated with intrascalar electrodes in either monopolar or bipolar configurations. Also, consistent with the distributions of limiting rates shown in Figure 4 , intraneural and apical ball electrodes activated the largest number of units having high limiting rates. Regardless of stimulus configuration, however, units with CFs $<1.5 \mathrm{kHz}$ tended to have the highest limiting rates, up to the highest rate tested, and most units with $\mathrm{CFs}>1.5 \mathrm{kHz}$ tended to have limiting rates below $\sim 400$ pps. That indicates that the paucity of units with high limiting rates observed with intrascalar stimulation likely is attributable to the small number of low-CF neurons that could be stimulated with intrascalar electrodes. In Figure $6 A$, the three units with $\mathrm{CFs}>16 \mathrm{kHz}$ and limiting rates of 600 pps are exceptions to the high-CF low-limiting-rate pattern. We note that those three units were distributed among three of the five cats, so they are not characteristic of a single aberrant experiment.

At the higher stimulus level, 2 or $4 \mathrm{~dB}$ above the minimum suprathreshold level, intraneural stimulation showed the same relationship of limiting rate to CF seen at lower levels. In the monopolar- and bipolar-stimulation conditions, stimulus levels 2 or $4 \mathrm{~dB}$ above threshold rarely were achieved for ICC units with CFs $<1.5 \mathrm{kHz}$. Most units with CFs $>1.5 \mathrm{kHz}$ had limiting rates $<400$ pps. Among the few units with $\mathrm{CFs}<1.5 \mathrm{kHz}$ that could be 


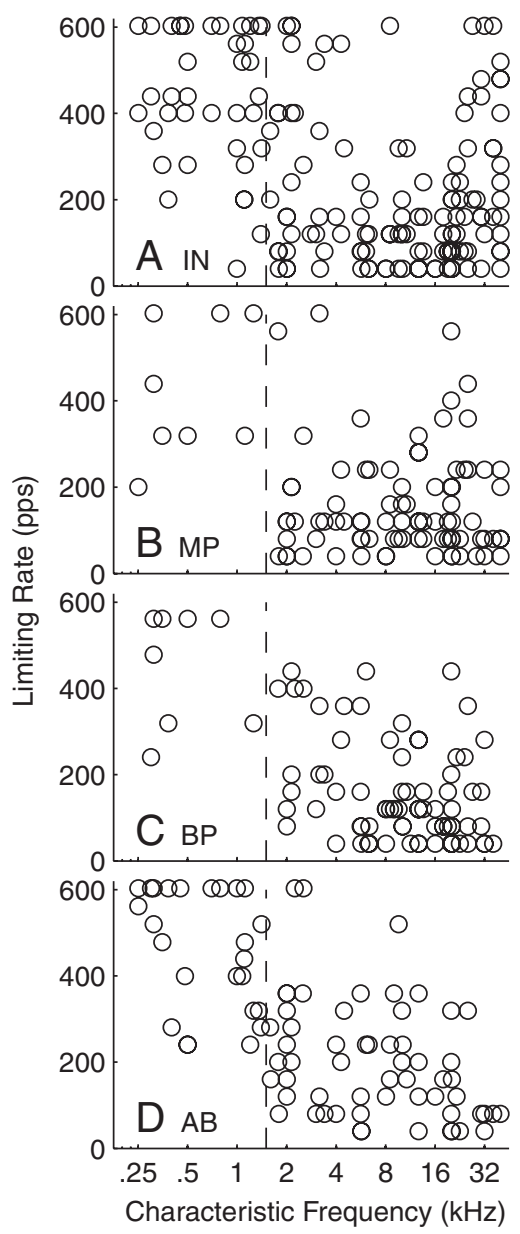

Figure 6. Limiting rates as a function of characteristic frequency. Each panel represents a particular electrode type or configuration, as indicated. IN, Intraneural; MP, monopolar intrascalar; BP, bipolar intrascalar; AB, apical ball.

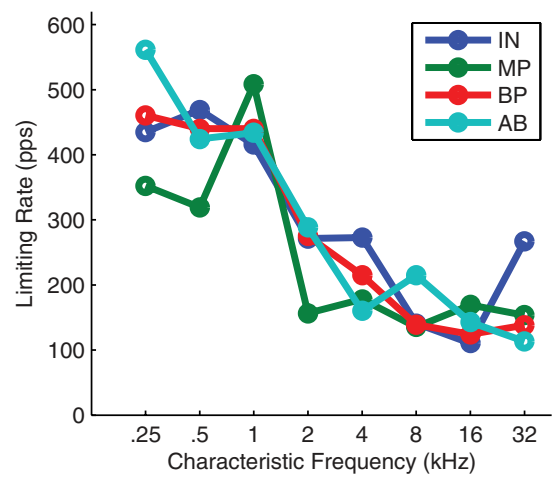

Figure 7. Limiting rates for various electrode types as a function of characteristic frequency, averaged by 1 octave blocks of frequency. IN, Intraneural; MP, monopolar intrascalar; BP, bipolar intrascalar; $\mathrm{AB}$, apical ball.

stimulated at intrascalar stimulus levels 2 or $4 \mathrm{~dB}$ above the minimum suprathreshold level, most had high limiting rates.

We evaluated the dependence of limiting rate on CF and electrode configuration using a two-way ANOVA. Figure 7 shows mean limiting rates grouped by configuration and by CF in 1 octave groups. The relationship between $\mathrm{CF}$ and limiting rate was significant for all stimulus configurations $(p<0.0001)$. After accounting for CF, there was no significant dependence of limit- ing rate on electrode configuration ( $p=0.25$ ), as indicated by the very similar trajectory of the lines in Figure 6 representing particular configurations. There are two instances in which limiting rates for a particular configuration deviated from those of other configurations. At CFs centered on 0.25 and $0.5 \mathrm{kHz}$, the limiting rates were lower for monopolar stimulation than for other configurations. That CF range was represented by very few units that could be activated in the monopolar configuration, so the reliability of those mean values is questionable. In the $32 \mathrm{kHz} C F$ group, the mean limiting rate for the intraneural condition was somewhat higher than expected. That mean data point includes the three anomalous high-CF, high-limiting-rate points noted in Figure $5 A$.

In cases in which a particular ICC unit could be activated by more than one stimulus configuration (e.g., by intraneural and by monopolar intrascalar), there was no significant difference in the limiting rate observed with the various configurations; ANOVAs showed $p>0.05$ for any two-way comparison of low-CF intraneural electrode, high-CF intraneural electrode, monopolar, bipolar, or apical ball. The correlation coefficients between all pairs of those configurations, however, were significant $(r=0.40-0.57, p<0.01$ to $p<0.0001)$ with the exception of correlations between the high-CF intraneural electrodes and the low-CF apical intraneural electrodes or the monopolar or bipolar intrascalar electrodes $(p>0.05)$. The lack of significant correlation in the last case likely was attributable to the low limited overlap of patterns of activation for those configurations, which resulted in large disparities in thresholds.

\section{First-spike latencies and group delays}

Previous studies of phase locking of ICC and cortical neurons to pulsatile or modulated stimuli have found that higher limiting rates tend to be associated with shorter first-spike latencies (Langner et al., 1987; Snyder et al., 1995; Middlebrooks, 2008). We tested the hypothesis that high limiting rates for ICC phase locking to fine structure of electrical pulse trains were associated with short first-spike latencies. Figure 8 shows evidence strongly supporting that hypothesis. For all electrode configurations, there was a significant relationship between limiting rate and first-spike latency $(p<0.0001)$. Units with limiting rates greater than $\sim 300$ pps tended to have latencies shorter than $6 \mathrm{~ms}$, and units with latencies longer than $6 \mathrm{~ms}$ tended to have limiting rates lower than 300 pps. A similar significant relationship between limiting rate and first-spike latency was observed at the higher stimulus level ( $p<0.0001$ for all conditions).

Group delay provided a measure of the ear-to-ICC delays in the tonic portions of ICC responses. Group delay was calculated from the slope of the line relating mean phase lag to stimulus pulse rate, as demonstrated in Figure 3 (see Materials and Methods). In the auditory cortex, group delays can be substantially longer than first-spike latencies, likely as a result of intracortical inhibition (Middlebrooks, 2008). In the present ICC dataset (data not illustrated), group delays tended to correlate with firstspike latencies ( $r=0.46-0.60$ depending on configuration; all $p<0.0001)$. The monopolar and bipolar intrascalar stimulation data showed no significant difference between first-spike latencies and group delays ( $t$ test, $p=0.18$ and 0.91 for monopolar and bipolar, respectively). In the cases of intraneural and apical ball stimulations, group delays averaged slightly shorter, 0.23 and $0.54 \mathrm{~ms}$, than first-spike latencies $(p<0.05$ and $p<0.001$ for intraneural and apical ball, respectively). Those results do not support the hypothesis that group delays are lengthened (or limiting rates are lowered) by intra-ICC mechanisms. A similar cor- 


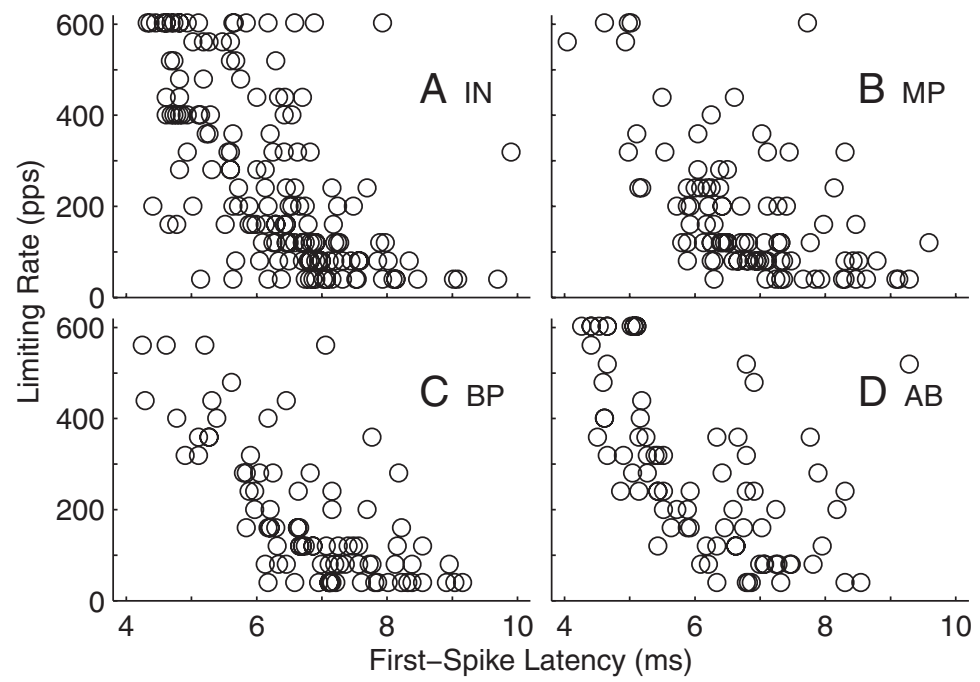

Figure 8. Limiting rate as a function of first-spike latency for electrical stimulation. Each panel represents a particular electrode type, as indicated. The relation of limiting rate to first-spike latency was significant for each electrode type ( $p<0.0001$, ANOVA). IN, Intraneural; MP, monopolar intrascalar; BP, bipolar intrascalar; AB, apical ball.

relation between group delay and first-spike latency was also observed at the higher stimulus level ( $p<0.0001$ to $p<0.001$ ). In that condition, group delays were significantly shorter than first-spike latencies only for the bipolar intraneural configuration ( $p<0.05$; mean difference, $0.45 \mathrm{~ms}$ ). Consistent with the relationship between limiting rates and first-spike latencies and with the correlation between group delays and first-spike latencies, there was a significant trend for units with high limiting rates to have short group delays (generally $<6 \mathrm{~ms}$ ) and for units with low limiting rates to have longer group delays $(>6 \mathrm{~ms})(p<0.0001$ for all stimulus configurations).

The observations that high limiting rates are associated with short first-spike latencies and with low CFs imply that short firstspike latencies for electrical stimulation are associated with low CF. That relationship is shown in Figure 9. The scatters of firstspike latencies within any particular range of CF were broad, but each stimulus configuration showed a significant increase in the first-spike latency for electrical stimulation associated with increasing CF (one-way ANOVA; $p<0.0001$ to $p<0.001$ for all configurations). The most conspicuous trend was between $\mathrm{CF}$ ranges below or above $\sim 1.5 \mathrm{kHz}$, with little consistent trend within low-CF or within high-CF ranges. The broad scatter of latencies among units with high CFs can be explained somewhat by the scatter in limiting rates within any particular range of CFs. Among units with $\mathrm{CF}>1.5 \mathrm{kHz}$, two-way ANOVA showed a significant main effect of limiting rate on first-spike latency $(p<$ 0.005 for the apical ball configuration; $p<0.0001$ for all other configurations). That shows that units with the shortest latencies tended to have the highest limiting rates, even after removing the CF dependence of latency.

At the higher stimulus levels, a short-to-long latency trend associated with increasing CF also was observed for intraneural stimulation $(p<0.0001)$. There was no significant trend for the other configurations at the high stimulus levels, most likely because of the limited ranges of CF activated by monopolar and bipolar stimulation (mostly $>1.5 \mathrm{kHz}$ ) and apical ball stimulation (mostly $<1.5 \mathrm{kHz}$ ).

As was true for limiting rates, first-spike latencies showed no significant difference between stimulation configurations in cases in which ICC units could be stimulated by two or more configurations. The difference in firstspike latencies was not significant for any pair of stimulus configurations. Pairwise correlations of latencies measured for various stimulus configurations were significant $(r=0.37-0.66 ; p<0.05$ to $p<$ $0.0001)$ with the exceptions of the high-CF intraneural stimulation compared with the low-CF intraneural stimulation or with monopolar stimulation $(p>0.05)$.

In conditions of acoustical stimulation, the cochlear traveling wave tends to delay responses to low-frequency sounds. For that reason, one would expect tonal stimulation to show a long-to-short-latency trend associated with increasing $\mathrm{CF}$ for units in the ICC, opposite to the short-to-long-latency trend observed with electrical stimulation. The expected direction of the latency trend for tones was confirmed in the present study (Fig. 10) ( $p<0.0005)$. It follows that first-spike latencies for electrical and acoustical stimulation were inversely correlated $(r=-0.29 ; p<0.005 ; n=102)$.

\section{Discussion}

\section{A low-CF, high-temporal-acuity brainstem pathway}

The present results demonstrate that the brainstem pathway from the cochlear apex to low-CF neurons in the ICC is characterized by shorter first-spike latencies, shorter group delays, and higher limiting rates for phase locking than are exhibited by highfrequency pathways. Those characteristics are consistent with an adaptation for preservation of auditory-nerve phase locking to the fine structure of low-frequency sounds. Conversely, we see no selective pressure for preservation of temporal fine structure in high-frequency brainstem pathways, because high-frequency nerve fibers do not phase lock to their best-frequency sounds.

We observed the greatest difference in latencies between unit populations having CFs above or below $1.5 \mathrm{kHz}$. The most likely anatomical substrate for a low-frequency, high-speed, hightemporal-acuity brainstem pathway is the one beginning in the large spherical bushy cells (SBCs) of the anterior ventral cochlear nucleus $(\mathrm{AVCN})$, projecting through the trapezoid body to the medial superior olive (MSO), and continuing to the lowfrequency isofrequency laminae of the ICC. Each SBC receives auditory-nerve input from the apical cochlea in the form of one to four (mostly two) of the distinctive axosomatic end bulbs of Held, which appear to be specialized for high-fidelity transmission of auditory-nerve spike timing (Osen, 1969; Cant, 1991; Ryugo and Sento, 1991; Joris and Smith, 2008). Joris et al. (1994) recorded from fibers in the trapezoid body presumed to be the axons of SBCs. Fibers with CFs $<1 \mathrm{kHz}$ showed phase locking to acoustical fine structure that was sharper than that observed in auditory nerve fibers. In contrast, trapezoid-body fibers with $\mathrm{CFs}>2 \mathrm{kHz}$ showed less precise phase locking than that in the auditory nerve. The large SBCs project bilaterally to principal cells of the MSO, which phase lock to low-frequency tonal stimuli (Goldberg and Brown, 1969; Yin and Chan, 1990). Those neurons project to the ICC, with terminations widely distributed across low-frequency isofrequency laminae (Oliver et al., 2003).

One might question how the SBC-to-MSO-to-ICC pathway could have shorter latencies than pathways that project directly from the cochlear nucleus to the ICC without an intervening 


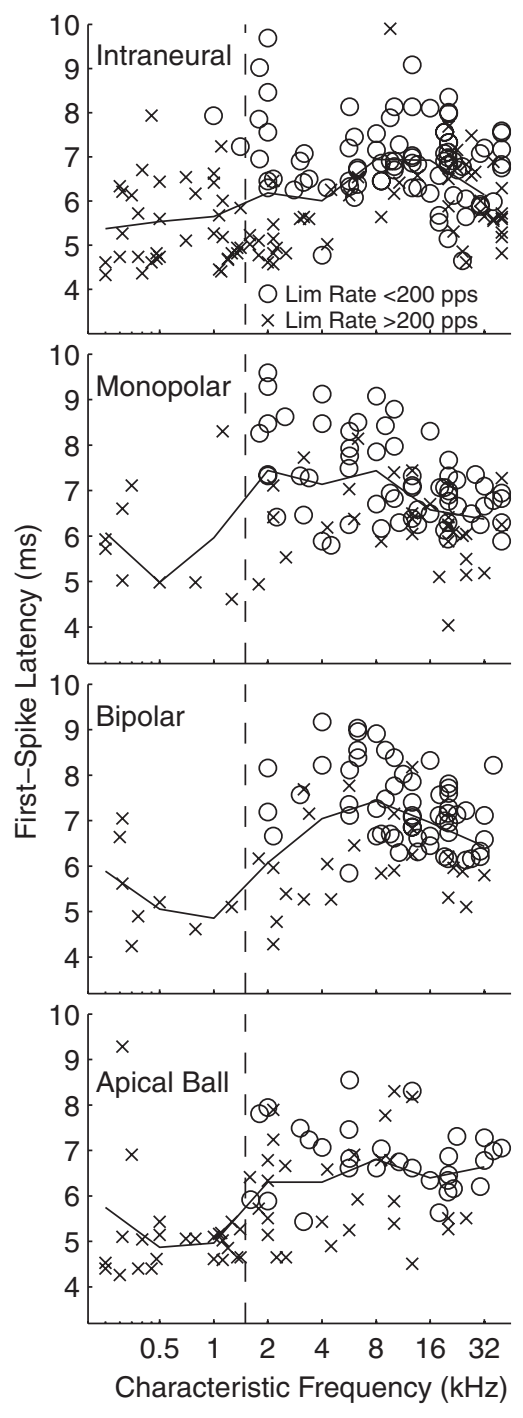

Figure 9. First-spike latency for electrical stimulation as a function of characteristic frequency. Each panel represents a particular electrode type, as indicated. The lines indicate averages of first-spike latency group in $10 c t a v e$ blocks of characteristic frequency. Latency increased significantly with increasing $\mathrm{CF}$ ( $p<0.0001$ to $p<0.001$, depending on the stimulating electrode type). Symbols indicate units with limiting rates above or below 200 pps, as indicated.

synapse. Of the cochlear-nucleus cell types that project directly to the ICC, however, none have high temporal acuity. The two major classes of large direct-projecting cells are as follows: type I stellate (or multipolar) cells in the posterior ventral cochlear nucleus (PVCN), which receive primarily small axodendritic auditory nerve terminals and which exhibit long-latency, periodic "chopper" responses that are unrelated to the stimulus period (Smith and Rhode, 1989; Cant and Benson, 2003); and fusiform (or pyramidal) cells of the dorsal cochlear nucleus, which have long first-spike latencies and temporally nonlinear responses to modulated stimuli (Joris and Smith, 1998). Globular bushy cells in the AVCN and octopus cells in the PVCN have short latencies and high temporal acuity, but they project to the ICC primarily by way of a synapse in the medial nucleus of the trapezoid body (globular bushy cells) or ventral nucleus of the trapezoid body (octopus cells), in both cases resulting in net inhibition to the contralateral ICC. That the high-temporal-acuity pathway is limited to low CFs presumably reflects the distribution of large SBCs amid the terminals of apical auditory nerve fibers in the anterior pole of the AVCN.

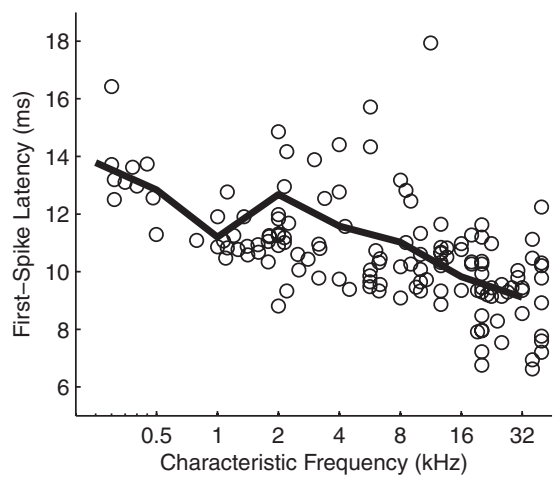

Figure 10. First-spike latency for tonal stimulation as a function of characteristic frequency. The line indicates averages of first-spike latency group in 1 octave blocks of characteristic frequency. First-spike latencies decreased significantly with increasing $\mathrm{CF}(p<0.0005)$.

The tendency of low-CF units in the ICC to show shorter latencies than higher-CF units under conditions of electrical auditory nerve stimulation is contrary to the trend observed with acoustical stimulation in the present and previous studies (Langner et al., 1987; Langner and Schreiner, 1988; Joris et al., 2006; Liu et al., 2006): sound-evoked latencies decreased by $>4$ ms over the 7 octave range of increasing CF that we tested. The latency trend observed with acoustic stimulation can be attributed primarily to the influence of the cochlear traveling wave, which can result in an $\sim 5 \mathrm{~ms}$ dispersion of high-to-low-CF auditory-nerve discharges (Kiang, 1965; Goldstein et al., 1971; Ruggero and Temchin, 2007). We note that Joris et al. (2006, their Fig. 11C) showed the sound-evoked latencies of ICC neurons steadily declining with $\mathrm{CF}$ increasing to $\sim 2 \mathrm{kHz}$, closely paralleling auditory-nerve latencies, but ICC latencies increasing across CFs from $\sim 2$ to $3 \mathrm{kHz}$, resulting in lengthening nerve-toICC delay. The reduced nerve-to-ICC delay of low-CF pathways revealed with electrical stimulation would serve to partially compensate for the traveling wave delay in normal-hearing conditions. Nevertheless, that compensation apparently is not complete, as evidenced by the across-CF dispersion of latencies recorded in the ICC in acoustic-stimulation conditions. Rising-frequency acoustic chirps can offset the frequency dispersion of the traveling wave and result in enhanced synchrony of auditory-nerve compound action potentials (Shore and Nuttall, 1985) and enhanced wave-V auditory brainstem responses (Dau et al., 2000). Surprisingly, in a human psychophysical experiment, rising-frequency chirps were reported to sound less temporally compact than simple acoustic clicks or even falling-frequency chirps (Uppenkamp et al., 2001). That suggests the existence of additional compensation between the temporally dispersed latencies in the ICC and the perception of temporal compactness.

We observed a robust inverse correlation between high-tolow limiting rates and short-to-long first-spike latencies and group delays. A more modest trend was reported previously from studies that used cochlear-implant stimulation and ICC recording (Snyder et al., 1995, 2000; Vollmer et al., 1999). The trend was more evident in the present study presumably because intraneural stimulation permitted us to sample a broader range of the tonotopic axis in the brainstem and, consequently, a broader range of latencies and limiting rates. Among ICC neurons with $\mathrm{CF}>1.5 \mathrm{kHz}$, units with limiting rates $>200$ pps had significantly shorter latencies than those with lower limiting rates, consistent with results obtained using amplitude-modulated highfrequency tones (Langner and Schreiner, 1988; Krishna and 
Semple, 2000; Nelson and Carney, 2007). In the auditory cortex, neurons exhibiting low limiting rates to amplitude-modulated cochlear-implant stimulation have group delays that are appreciably longer than their first-spike latencies, which suggests the presence of an intracortical low-pass filter (Eggermont, 1999; Middlebrooks, 2008). The present ICC results showed no such difference between group delays and first-spike latencies. For that reason, we hypothesize that the relationship between first-spike latency and limiting rate observed in the ICC reflects properties of brainstem pathways rather than intra-ICC mechanisms.

\section{Fine-structure sensitivity in human cochlear-implant users} Cochlear-implant users show impaired sensitivity to temporal fine structure compared with normal-hearing listeners. In tests of "rate pitch," implant users can detect $\sim 10 \%$ changes in the rates of pulse trains below $\sim 200-300$ pps, but rate sensitivity is lost at higher rates (Shannon, 1983; Tong and Clark, 1985; Townshend et al., 1987; McKay et al., 2000; Zeng, 2002; Landsberger and McKay, 2005; van Hoesel, 2007; Carlyon et al., 2008). In contrast, normal-hearing listeners can discriminate pure tone frequencies with difference limens of $\sim 1 \%$ up to frequencies of $\sim 2000 \mathrm{~Hz}$. Moreover, at low pulse rates, bilateral cochlear-implant users show thresholds for interaural time differences that are comparable with those of normal-hearing listeners, whereas that sensitivity is entirely lost at pulse rates $>100$ pps (van Hoesel and Tyler, 2003; van Hoesel, 2007).

At least part of the impairment in temporal fine-structure sensitivity in cochlear-implant users could be attributable to a failure to stimulate low-frequency brainstem pathways specialized for preservation of temporal fine structure. Most cochlear implant electrodes are located in the cochlear base and stimulate primarily the high-frequency pathways originating there. It is doubtful that even the most apical intrascalar electrodes achieve selective stimulation of apical fibers, either because of the compact geometry of the spiral ganglion in the cochlear apex (Baumann and Nobbe, 2004, 2006; Stakhovskaya et al., 2007) or because of unintended deviation of apical electrodes into the scala vestibuli (Skinner et al., 2007; Finley et al., 2008). In cases in which excitation spreads to apical fibers from more basal electrodes, perception of periodicity through low-frequency pathways might be masked by nonsynchronized activity in high-frequency pathways stimulated at higher sensation levels. In a future auditory prosthesis using intraneural stimulation, one might expect to achieve frequency-specific stimulation of low-CF pathways that could lead to improved sensitivity to temporal fine structure. Although we have no results from bilateral intraneural stimulation, we also speculate that selective stimulation of low-frequency pathways could enhance sensitivity to interaural time differences.

Failure to activate low-frequency pathways cannot account entirely for the impaired temporal acuity of implant users. When bandpass harmonic complexes were used to test temporal pitch sensitivity in the basal cochlea, normal hearing listeners showed temporal pitch perception up to $\sim 600$ pps compared with $\sim 300$ pps for cochlear-implant users (Carlyon and Deeks, 2002; Carlyon et al., 2008). At least in the basal cochlea, therefore, temporal acuity of implant users might also be impaired to some degree by characteristics of the auditory nerve, such as partial loss of nerve fibers or by the absence of spontaneous activity. It remains to be tested how well temporal sensitivity in electric hearing might be improved by selective stimulation of apical-turn fibers.

\section{References}

Baumann U, Nobbe A (2004) Pulse rate discrimination with deeply inserted electrode arrays. Hear Res 196:49-57.

Baumann U, Nobbe A (2006) The cochlear implant electrode-pitch function. Hear Res 213:34-42.

Cant NB (1991) Projections to the lateral and medial superior olivary nuclei from the spherical and globular bushy cells of the anteroventral cochlear nucleus. In: Neurobiology of hearing: the central auditory system (Altschuler RA, Bobbin RP, Clopton BM, Hoffman DW, eds), pp 99-119. New York: Raven.

Cant NB, Benson CG (2003) Parallel auditory pathways: projection patterns of the different neuronal populations in the dorsal and ventral cochlear nuclei. Brain Res Bull 60:457-474.

Carlyon RP, Deeks JM (2002) Limitations of rate discrimination. J Acoust Soc Am 112:1009-1025.

Carlyon RP, Long CJ, Deeks JM (2008) Pulse-rate discrimination by cochlear-implant and normal-hearing listeners with and without binaural cues. J Acoust Soc Am 123:2276-2286.

Dau T, Wegner O, Mellert V, Kollmeier B (2000) Auditory brainstem responses with optimized chirp signals compensating basilar-membrane dispersion. J Acoust Soc Am 107:1530-1540.

Eggermont JJ (1999) The magnitude and phase of temporal modulation transfer functions in cat auditory cortex. J Neurosci 19:2780-2788.

Finley CC, Holden TA, Holden LK, Whiting BR, Chole RA, Neely GJ, Hullar TE, Skinner MW (2008) Role of electrode placement as a contributor to variability in cochlear implant outcomes. Otol Neurotol 29:920-928.

Goldberg JM, Brown PB (1969) Response of binaural neurons of dog superior olivary complex to dichotic tonal stimuli: some physiological mechanisms of sound localization. J Neurophysiol 32:613-636.

Goldstein JL, Baer T, Kiang NYS (1971) A theoretical treatment of latency, group delay, and tuning characteristics for auditory nerve responses to clicks and tones. In: The physiology of the auditory system (Sachs MB, ed), pp 133-141. Baltimore: National Educational Consultants.

Green DM, Swets JA (1966) Signal detection theory and psychophysics. New York: Wiley.

Hartmann R, Klinke R (1990) Response characteristics of nerve fibers to patterned electrical stimulation. In: Cochlear implants, models of the electrically stimulated ear (Miller JM, Spelman FA, eds), pp 136-160. New York: Springer.

Javel E (1990) Acoustical and electrical encoding of temporal information. In: Cochlear implants, models of the electrically stimulated ear (Miller JM, Spelman FA, eds), pp 247-291. New York: Springer.

Joris PX, Smith PH (1998) Temporal and binaural properties in dorsal cochlear nucleus and its output tract. J Neurosci 18:10157-10170.

Joris PX, Smith PH (2008) The volley theory and the spherical cell puzzle. Neuroscience 154:65-76.

Joris PX, Carney LH, Smith PH, Yin TC (1994) Enhancement of synchronization in the anteroventral cochlear nucleus. J Neurophysiol 71:1022-1036.

Joris PX, van de Sande B, Recio-Spinoso A, van der Heijden M (2006) Auditory midbrain and nerve responses to sinusoidal variations in interaural correlation. J Neurosci 26:279-289.

Kiang NYS (1965) Discharge patterns of single fibers in the cat's auditory nerve. Cambridge, MA: Massachusetts Institute of Technology.

Krishna BS, Semple MN (2000) Auditory temporal processing: Responses to sinusoidally amplitude-modulated tones in the inferior colliculus. J Neurophysiol 84:255-273.

Landsberger DM, McKay CM (2005) Perceptual differences between low and high rates of stimulation on single electrodes for cochlear implantees. J Acoust Soc Am 117:319-327.

Langner G, Schreiner CE (1988) Periodicity coding in the inferior colliculus of the cat. I. Neuronal mechanisms. J Neurophysiol 60:1799-1822.

Langner G, Schreiner C, Merzenich MM (1987) Covariation of latency and temporal resolution in the inferior colliculus of the cat. Hear Res 31:197-201.

Liu LF, Palmer AR, Wallace MN (2006) Phase-locked responses to pure tones in the inferior colliculus. J Neurophysiol 95:1926-1935.

Macmillan NA, Creelman CD (2005) Detection theory: a user's guide, Ed 2. Mahwah, NJ: Erlbaum.

Mardia KV (1972) Statistics of directional data. London: Academic.

McKay CM, McDermott HJ, Carlyon RP (2000) Place and temporal cues in 
pitch perception: are they truly independent? Acoust Res Let Online $1: 25-30$.

Middlebrooks JC (2008) Auditory cortex phase locking to amplitudemodulated cochlear implant pulse trains. J Neurophysiol 100:76-91.

Middlebrooks JC, Snyder RL (2007) Auditory prosthesis with a penetrating nerve array. J Assoc Res Otolaryngol 8:258-279.

Middlebrooks JC, Snyder RL (2008) Intraneural stimulation for auditory prosthesis: modiolar trunk and intracranial stimulation sites. Hear Res 242:52-63.

Moore BCJ (1973) Frequency difference limens for short-duration tones. J Acoust Soc Am 54:610-619.

Nelson PC, Carney LH (2007) Neural rate and timing cues for detection and discrimination of amplitude-modulated tones in the awake rabbit inferior colliculus. J Neurophysiol 97:522-539.

Oliver DL, Beckius GE, Bishop DC, Loftus WC, Batra R (2003) Topography of interaural temporal disparity coding in projections of medial superior olive to inferior colliculus. J Neurosci 23:7438-7449.

Osen KK (1969) Cytoarchitecture of the cochlear nuclei in the cat. J Comp Neurol 136:453-484.

Ruggero MA, Temchin AN (2007) Similarity of traveling-wave delays in the hearing organs of humans and other tetrapods. J Assoc Res Otolaryngol 8:153-166.

Ryugo DK, Sento S (1991) Synaptic connections of the auditory nerve in cats: relationship between endbulbs of Held and spherical bushy cells. J Comp Neurol 305:35-48.

Shannon RV (1983) Multichannel electrical stimulation of the auditory nerve in man. I. Basic psychophysics. Hear Res 11:157-189.

Shore SE, Nuttall AL (1985) High synchrony compound action potentials evoked by rising frequency-swept tonebursts. J Acoust Soc Am 78:12861295.

Skinner MW, Holden TA, Whiting BR, Voie AH, Brunsden B, Neely JG, Saxon EA, Hullar TE, Finley CC (2007) In vivo estimates of the position of advanced bionics electrode arrays in the human cochlea. Ann Otol Rhinol Laryngol Suppl 197:2-24.

Smith PH, Rhode WS (1989) Structural and functional properties distin- guish two types of multipolar cells in the ventral cochlear nucleus. J Comp Neurol 282:595-616.

Snyder R, Leake P, Rebscher S, Beitel R (1995) Temporal resolution of neurons in cat inferior colliculus to intracochlear electrical stimulation: effects of neonatal deafening and chronic stimulation. J Neurophysiol 73:449-467.

Snyder RL, Vollmer M, Moore CM, Rebscher SJ, Leake PA, Beitel RE (2000) Responses of inferior colliculus neurons to amplitude-modulated intracochlear electrical pulses in deaf cats. J Neurophysiol 84:166-183.

Stakhovskaya O, Sridhar D, Bonham BH, Leake PA (2007) Frequency map for the human cochlear spiral ganglion: implications for cochlear implants. J Assoc Res Otolaryngol 8:220-233.

Tong YC, Clark GM (1985) Absolute identification of electric pulse rates and electrode positions by cochlear implant patients. J Acoust Soc Am 77:1881-1888.

Townshend B, Cotter N, Van Compernolle D, White RL (1987) Pitch perception by cochlear implant subjects. J Acoust Soc Am 82:106-115.

Uppenkamp S, Fobel S, Patterson RD (2001) The effects of temporal asymmetry on the detection and perception of short chirps. Hear Res 158:71-83.

van den Honert C, Stypulkowski PH (1987) Temporal response patterns of single auditory nerve fibers elicited by periodic electrical stimuli. Hear Res 29:207-222.

van Hoesel RJ (2007) Sensitivity to binaural timing in bilateral cochlear implant users. J Acoust Soc Am 121:2192-2206.

van Hoesel RJ, Tyler RS (2003) Speech perception, localization, and lateralization with bilateral cochlear implants. J Acoust Soc Am 113:1617-1630.

Vollmer M, Snyder RL, Leake PA, Beitel RE, Moore CM, Rebscher SJ (1999) Temporal properties of chronic cochlear electrical stimulation determine temporal resolution of neurons in cat inferior colliculus. J Neurophysiol 82:2883-2902.

Yin TC, Chan JC (1990) Interaural time sensitivity in medial superior olive of cat. J Neurophysiol 64:465-488.

Zeng FG (2002) Temporal pitch in electric hearing. Hear Res 174:101-106. 